\title{
CONTINUOUS VERSUS ON-DEMAND PROTON PUMP INHIBITOR TREATMENT FOR LARYNGOPHARYNGEAL REFLUX: A RANDOMIZED CLINICAL TRIAL
}

\author{
SUSYANA TAMIN ${ }^{*}$, ABDUL AZIZ RANI², ADANG BACHTIAR ${ }^{3}$
}

${ }_{1}^{1}$ Department of Otorhinolaryngology-Head and Neck Surgery, Faculty of Medicine, University of Indonesia-Dr. Cipto Mangunkusumo Hospital, Jakarta, Indonesia, ${ }^{2}$ Division of Gastroenterohepatology, Department of Internal Medicine, Faculty of Medicine, University of Indonesia-Dr. Cipto Mangunkusumo Hospital, Jakarta, Indonesia, ${ }^{3}$ Department of Health Policy and Administration, Faculty of Public Health, University of Indonesia, Jakarta, Indonesia

Email: usyana@yahoo.com

Received: 10 Dec 2018, Revised and Accepted: 10 Mar 2019

\section{ABSTRACT}

Objective: This study aimed to compare continuous and pro re nata (PRN) proton pump inhibitor (PPI) treatments for laryngopharyngeal reflux (LPR).

Methods: This randomized clinical trial included 52 LPR patients with lingual tonsil hypertrophy (LTH). Those patients who showed LPR improvement with an initial PPI trial were randomly divided into continuous and PRN PPI treatment groups. The Reflux Symptom Index (RSI) score, Reflux Finding Score (RFS), and LTH grade were used to assess the treatment results.

Results: The PPI treatment (30 mg of lansoprazole twice daily) for 6 mo resulted in a significantly decreasing RSI score (p<0.001). The PPI treatments for the first $8 \mathrm{w}$ and the second $8 \mathrm{w}$ also showed significant LPR improvement. However, there was no significant improvement after the third $8 \mathrm{w}(\mathrm{p}>0.05)$. After $6 \mathrm{mo}$, the PPI treatment also resulted in a continuous decrease in the RFS $(\mathrm{p}<0.001)$. There were no significant differences in the mean RSI scores $(\mathrm{p}=0.518)$ and mean RFSs $(\mathrm{p}=0.393)$ between the continuous and PRN PPI groups. In the grade II LTH cases, there was a significant improvement after 6 mo of PPI treatment $(p<0.001)$, although there was no improvement after the first 2 mo in the treatment group. For the grade III LTH cases, there was no significant improvement after the first $2 \mathrm{mo}$ and $6 \mathrm{mo}$ of the PPI treatment.

Conclusion: Six months of PPI treatment improved the RSI score, RFS, and LTH grade, although there was no difference between the continuous and PRN PPI treatment groups.

Keywords: Laryngopharyngeal reflux, Proton pump inhibitor, Continuous, Pro re nata treatment

(c) 2019 The Authors. Published by Innovare Academic Sciences Pvt Ltd. This is an open access article under the CC BY license (http://creativecommons. org/licenses/by/4. 0/) DOI: http://dx.doi.org/10.22159/ijap.2019.v11s6.33530

\section{INTRODUCTION}

Laryngopharyngeal reflux (LPR) disease is the backflow of extraesophageal gastric fluid into the larynx, pharynx, trachea, and bronchus, which may lead to various symptoms, such as a dry cough, globus sensation, dysphonia, throat clearing, postnasal drip, and dysphagia [1-3]. The LPR symptoms are different from typical gastroesophageal reflux disease (GERD) symptoms, which include esophagitis [1, 2]. Belafsky et al. developed a 9-item Reflux Symptom Index (RSI) and an 8-item Reflux Finding Score (RFS) for the evaluation of laryngeal abnormalities caused by LPR $[4,5]$.

Empirical therapy using proton-pump inhibitors (PPIs) for both diagnostic testing and LPRD and GERD treatment has become widely accepted [6]. Not only are PPIs noninvasive and simple to use, but they can also provide a rational and reasonable value to the treatment of these patients. Until now, there have been no studies examining the PPI treatment efficacy in LPRD patients. Therefore, this study was designed to ascertain the responses to continuous or pro re nata (PRN) PPI treatment based on the LPR improvement using the RSI and RFS results and the lingual tonsil hypertrophy (LTH) degree.

\section{MATERIALS AND METHODS}

This study included 83 dysphagia patients presenting to the Dysphagia Clinic of the Otorhinolaryngology-Head and Neck Surgery, Faculty of Medicine, University of Indonesia-Dr. Cipto Mangunkusumo Hospital in Jakarta, Indonesia. The medical histories of these patients were obtained, as well as the RSI, RFS, general ear, nose, and throat examination, and fiberoptic nasopharyngolaryngoscopy examination results in order to determine the LTH and LPR degrees. The ethical approval for this study was granted by the Ethics Committee of the Faculty of Medicine at the University of Indonesia.

All of the patients were informed about the procedures involved in this research both verbally and in writing, and they all signed consent forms. After obtaining written consent, each patient was given $30 \mathrm{mg}$ of lansoprazole twice daily to establish an early diagnosis and as reflux therapy. Of the 83 patients, 19 were not involved in the randomization because they did not return after this research stage. For those patients who returned, if there was symptom improvement, the PPI regimen continued for 2 mo. After this, the patients were randomly divided into two groups: a continuous treatment group and a PRN treatment group. Each of the patients in the continuous treatment group was maintained on the $30 \mathrm{mg}$ of lansoprazole twice daily treatment regimen. However, the patients in the PRN group were given $30 \mathrm{mg}$ of lansoprazole twice daily only when they complained of symptoms, and the duration of the treatment was self-determined by each patient. Of the 64 remaining patients who participated in this study, 7 patients and 5 patients were lost to follow-up in fourth and sixth months, respectively; therefore, 52 patients participated through the end of this study.

The data analysis was conducted using IBM SPSS Statistics for Windows version 23.0 (IBM Corp., Armonk, NY, USA). A univariate analysis was used for descriptive statistics. A repeated analysis of variance hypothesis test for 2 independent groups was used for the nominal independent variables (continuous PPI and PRN PPI) and the numerical dependent variables.

\section{RESULTS}

In total, 52 patients completed this study: 25 in the continuous group and 27 in the PRN group. Thirty-five of these patients (67.3\%) were females and $17(32.7 \%)$ were males. The number of patients in the $40-69$ y old age group (59.6\%) was greater than that in the $20-$ $39 \mathrm{y}$ old age group $(40.4 \%)$. More than one half of the total patients $(55.7 \%)$ were in the moderate economic level category; $25 \%$ were in the higher economic level category, and $19.3 \%$ were in the lower economic level category. There were no significant differences between the continuous PPI and PRN PPI patient groups with regard 
to the sex, age, and economic level.

After 6 mo of PPI treatment, the patients showed improvements in their symptom complaints, clinical signs, RSI scores, RFSs, and LTH degrees. However, there were no significant differences between the improvements in the mean RSI score $(p=0.518)$ and mean RFS $(p=$ 0.393 ) between the continuous PPI group and the PRN PPI group. The 6-month RSI results for the continuous PPI and PRN PPI groups can be seen in fig. 1.

After the first $8 \mathrm{w}$, the PPI treatment results showed statistically significant improvements in the patients' complaints. The mean RS score after the first eight weeks of the PPI treatment improved from $21.83 \pm 6.80$ to $7.88 \pm 5.19(\mathrm{p}<0.001)$, and after the second $8 \mathrm{w}$ of treatment, the mean score further improved to $4.23 \pm 2.65(\mathrm{p}<0.001)$ However, the additional $8 \mathrm{w}$ of PPI treatment did not significantly improve the mean RSI score $(2.96 \pm 2.35, \mathrm{p}>0.05)$. There was a significant difference between the mean RSI score before treatment and the mean score after 6 mo of PPI treatment $(\mathrm{p}<0.001)$, but there was no significant difference in the mean RSI score between the continuous treatment group and the PRN treatment group $(\mathrm{p}=0.518)$ (fig. 1).

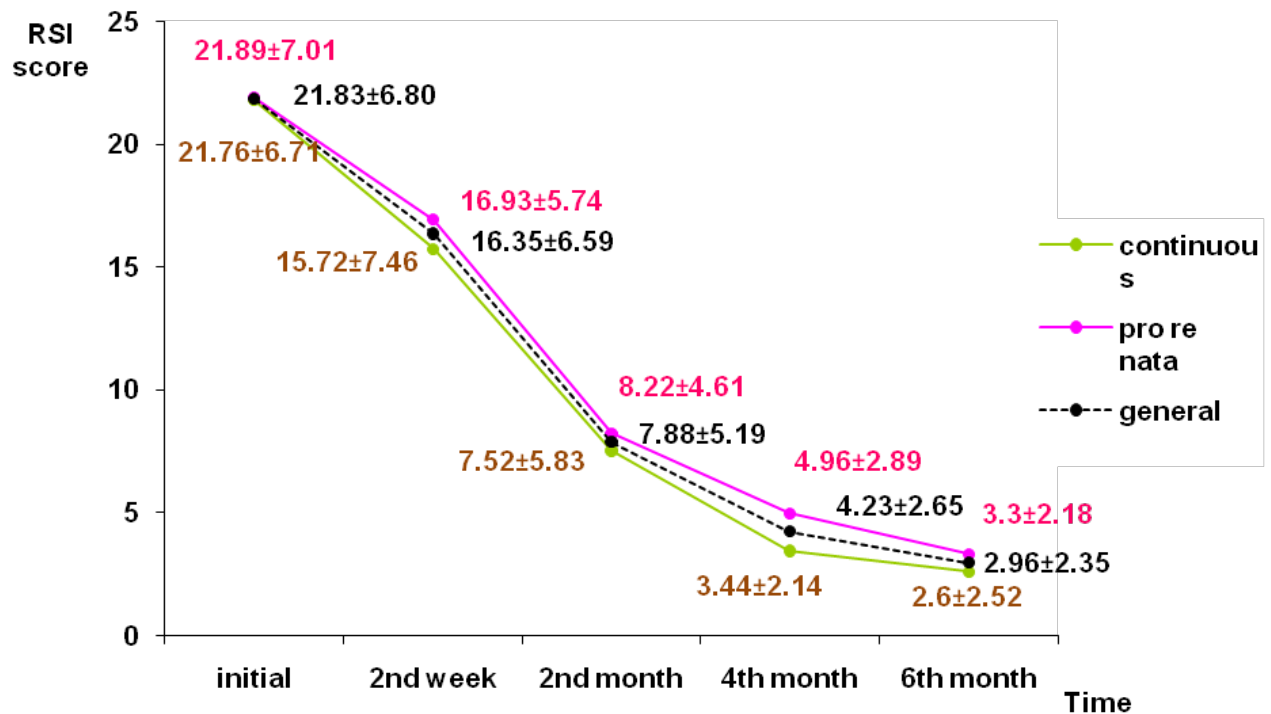

Legends: RSI: reflux symptom index PPI: proton pump inhibitor

Fig. 1: RSI score of continuous and pro re nata (PRN) PPI treatment groups in the following six months

Both the continuous and PRN PPI treatments improved the clinical signs in the laryngeal area. As shown in fig. 2, there was an improvement in the mean RFS score (from $10.98 \pm 2.52$ to $7.97 \pm 2.45$ ) after the first $8 \mathrm{w}$ of treatment $(\mathrm{p}<0.001)$. The mean RFS score improved again after the second $8 \mathrm{w}$ of treatment to $6.54 \pm 2.01(\mathrm{p}<0.001)$ and again to $4.73 \pm 1.95$ $(p<0.001)$ after the third $8 \mathrm{w}$ of treatment. The mean RFS scores slowly and continuously improved along with an improvement in the laryngeal area clinical signs through the 6 mo of PPI treatment. However, there was no significant difference between the continuous PPI group and the PRN PPI group ( $\mathrm{p}=0.393$ ) (fig. 2).

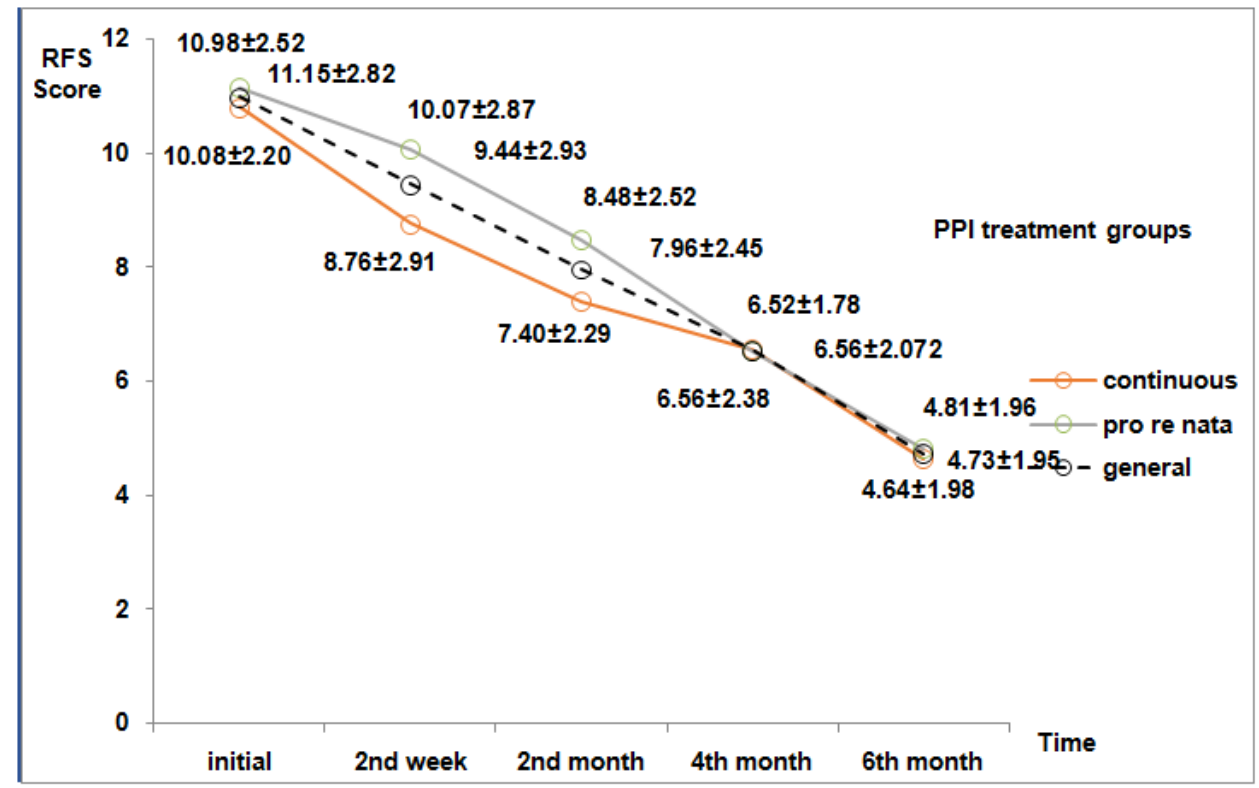

Legends: -RFS: reflux finding score,-PPI: proton pump inhibitor

Fig. 2: RFS score of continuous and pro re nata (PRN) PPI treatment groups in the following six months 
As shown in Tables 1 and 2, 2 mo of the twice-a-day continuous PPI dosing resulted in grade improvements, from LTH grade III to grade II and from LTH grade III to grade I in $44.1 \%$ and $8.8 \%$ of the patients, respectively. Although there were no grade improvements after 2 mo of the PPI treatment, there were grade improvements to
LTH grade I in $16.7 \%$ of patients in the continuous PPI group and $11.1 \%$ of the patients in the PRN PPI group after continuing the treatment for 4 mo. However, there were no further improvements after 6 mo of PPI treatment in either the continuous PPI group or the PRN PPI group.

Table 1: Lingual tonsil hypertrophy grading at the initial assessment, the second week of treatment, fourth week of treatment, and eighth week of treatment

\begin{tabular}{|c|c|c|c|c|c|}
\hline & \multirow[t]{2}{*}{ Initial grade of lingual tonsil hypertrophy } & \multicolumn{4}{|c|}{ Post PPI therapy of lingual tonsil hypertrophy } \\
\hline & & $1(n / \%)$ & $2(n / \%)$ & $3(n / \%)$ & Total (n/\%) \\
\hline \multirow[t]{4}{*}{ Second week } & 1 & $10(100)$ & $0(0)$ & $0(0)$ & $10(100)$ \\
\hline & 2 & $0(0)$ & $33(100)$ & $0(0)$ & $33(100)$ \\
\hline & 3 & $0(0)$ & $0(0)$ & $40(100)$ & $40(100)$ \\
\hline & Total & $10(12.0)$ & $33(39.8)$ & $40(48.2)$ & $83(100)$ \\
\hline \multirow[t]{4}{*}{ Fourth week } & 1 & $7(100)$ & $0(0)$ & $0(0)$ & $7(100)$ \\
\hline & 2 & $5(20.0)$ & $20(80)$ & $0(0)$ & $25(100)$ \\
\hline & 3 & $0(0)$ & $10(29.4)$ & $24(70.6)$ & $34(100)$ \\
\hline & Total & $12(18.2)$ & $30(45.5)$ & $24(36.4)$ & $66(100)$ \\
\hline \multirow[t]{4}{*}{ Eight week } & 1 & $7(100)$ & $0(0)$ & $0(0)$ & $7(100)$ \\
\hline & 2 & 12 (52.2) & $11(47.8)$ & $0(0)$ & $23(100)$ \\
\hline & 3 & $3(8.8)$ & $15(44.1)$ & $16(47.1)$ & $34(100)$ \\
\hline & Total & $22(34.4)$ & $26(40.6)$ & $16(25)$ & $64(100)$ \\
\hline
\end{tabular}

Table 2: Lingual tonsil hypertrophy grading after the fourth month of treatment and the sixth month of treatment

\begin{tabular}{|c|c|c|c|c|c|c|c|}
\hline & \multirow[t]{2}{*}{ After-eight-week treatment grade } & \multicolumn{6}{|c|}{ Post PPI treatment grade of lingual tonsil hypertrophy } \\
\hline & & & $1(n / \%)$ & $2(n / \%)$ & $3(n / \%)$ & Total (n/\%) & $\mathbf{p}$ \\
\hline \multirow[t]{9}{*}{ Fourth month } & 1 & continuous & $11(100)$ & $0(0)$ & $0(0)$ & $11(100)$ & \\
\hline & & pro re nata & $8(100)$ & $0(0)$ & $0(0)$ & $8(100)$ & \\
\hline & Total & & $19(100)$ & $0(0)$ & $0(0)$ & $19(100)$ & \\
\hline & 2 & continuous & $6(54.5)$ & $5(45.5)$ & $0(0)$ & $11(100)$ & 0.024 \\
\hline & & pro re nata & $1(8.3)$ & 11 (91.7) & $0(0)$ & $12(100)$ & \\
\hline & Total & & $7(30.4)$ & $16(69.6)$ & $0(0)$ & $23(100)$ & \\
\hline & 3 & continuous & $1(16.7)$ & $2(33.3)$ & $3(50.0)$ & $6(100)$ & 0.969 \\
\hline & & pro re nata & 1 (11.1) & 7 (77.8) & 1 (11.1) & $9(100)$ & \\
\hline & Total & & $2(13.3)$ & $9(60.0)$ & $4(26.7)$ & $15(100)$ & \\
\hline \multirow[t]{9}{*}{ Sixth month } & 1 & continuous & $8(100)$ & $0(0)$ & $0(0)$ & $8(100)$ & \\
\hline & & pro re nata & $7(100)$ & $0(0)$ & $0(0)$ & 7 (100) & \\
\hline & Total & & $15(100)$ & $0(0)$ & $0(0)$ & $15(100)$ & \\
\hline & 2 & continuous & 8 (72.7) & $3(27.3)$ & $0(0)$ & $11(100)$ & 0.01 \\
\hline & & pro re nata & $2(18.2)$ & $9(81.8)$ & $0(0)$ & $11(100)$ & \\
\hline & Total & & $10(45.5)$ & $12(54.5)$ & $0(0)$ & $22(100)$ & \\
\hline & 3 & continuous & $1(16.7)$ & $5(83.3)$ & $0(0)$ & $6(100)$ & 0.696 \\
\hline & & pro re nata & 1 (11.1) & $7(77.8)$ & 1 (11.1) & $9(100)$ & \\
\hline & Total & & $2(13.3)$ & $12(80.0)$ & $1(6.7)$ & $15(100)$ & \\
\hline
\end{tabular}

In the LTH grade II patients, there were improvements to LTH grade I in $52.2 \%$ of the patients after 2 mo of the PPI treatment. However, even though the condition remained the same (LTH grade II) after 2 mo of the PPI treatment, the continuous PPI treatment provided better results (72.7\%) than the PRN PPI treatment (18.2\%).

There was a significantly different LTH degree improvement between the two groups after 6 mo of treatment $(\mathrm{p}<0.001)$. Moreover, the continuous PPI group showed better overall improvement when compared to the PRN PPI group, although the difference was not significant $(p=0.158)$.

\section{DISCUSSION}

In LPR cases, the reliability of $24 \mathrm{~h} \mathrm{pH}$ monitoring has been questioned because it has poor sensitivity, poor specificity, and poor positive and negative predictive values [7]. Therefore, efforts have been made to improve the clinical examination reliability in order to identify LPR patients. Many researchers have used the RSI created by Belafsky et al., which is a 9-item, self-administered, disease- specific outcome instrument based on LPR symptoms. The item scores range from 0 (no complaints) to 5 (heavy sigh), with a maximum score of 45 , and RSI values $>13$ are considered to be abnormal [4]. Belafsky et al. also created the RFS, which is an 8-item clinical severity scale based on a patient's videolaryngoscopic findings. The scores range from 0 (no abnormal findings) to a maximum of 26 (worst possible score) $[5,8]$. Generally, an LPR diagnosis is made using a combination of the patient's history, symptoms, and endoscopic assessment results. Additionally, Campagnolo et al. suggested the use of a therapeutic PPI trial as a cost-effective and useful diagnostic test [2, 9]. The results of our study showed significant RSI score improvements with 6 mo of PPI treatment (from $21.83 \pm 6.80$ to $2.96 \pm 2.35$ ). This indicates that 6 mo of PPI treatment improved the patients' symptoms as assessed by the RSI score. Habermann et al. [10] also found RSI score improvements in their study after $12 \mathrm{w}$ of treatment with $40 \mathrm{mg}$ of pantoprazole daily. The median total RSI score before treatment was 12 (range=10-41), which decreased significantly to 3 (range $=0-44$ ) at the end of the treatment $(p<0.001)[10]$. In our study, there were 
significant improvements after 2 and 4 mo of the PPI treatment in both groups. However, the complaints remained the same, despite further therapy, up to the sixth month. These complaints could be further improved with dietary and lifestyle modifications, such as smoking cessation, weight loss, avoiding caffeine, and head-of-thebed elevation [11]. Pearson et al. [12]. Reported that dietary and behavior changes were very effective for the management of LPR in a UK district general hospital.

Sataloff et al. [13] had findings consistent with the first observation by Aviv in 1993 using the Flexible Endoscopic Evaluation of Swallowing with Sensory Testing, in which they found diminished sensory thresholds in the LPR patients. We used the RFS to assess the laryngeal area abnormalities, and our results showed that the mean RFS before treatment was $10.98 \pm 2.5$. There were significant improvements after 2 mo (7.96 \pm 2.4$), 4$ mo $(6.54 \pm 2.1)$, and 6 mo $(4.73 \pm 1.9)$ of PPI treatment $(\mathrm{p}<0.05)$. Our results also showed that there was no significant difference in the mean RFSs between the continuous PPI group and the PRN PPI group. However, the results did show that the PPI treatment improved the RFS with both the continuous and PRN PPI treatments. The results of the study by Patigaroo et al.[14] showed that the mean RFS before the PPI treatment in 50 patients was 12 , and that the most common laryngeal findings were laryngeal hyperemia, ventricular obliteration, and posterior commissure hypertrophy. The mean RFSs after $8 \mathrm{w}$ and $16 \mathrm{w}$ of the PPI treatment decreased significantly to 9.5 and 6.5 , respectively [14].

The RFS was designed to be an objective scoring system used to assess laryngeal area abnormalities. It has also been used to monitor the LPR progression and response to treatment. However, it is still unclear whether the results of both the RSI and RSF can be used to guide the treatment of suspected LPR $[3,10]$.

No previous studies have reported the effects of PPI treatment on LTH. In our study, there were LTH grade improvements that narrowed significantly after 6 mo of PPI treatment $(p<0.001)$, although the difference between the continuous and PRN PPI groups was not significant $(\mathrm{p}=0.158)$.

If we were to compare the outcomes from each baseline grade, not all of LTH grades would improve with PPI treatment. If there is no improvement in an LTH grade III patient after 2 mo of PPI treatment, continuing the PPI treatment up to 6 mo might only provide a little improvement. Therefore, other treatment options should be considered. Contrarily, when there is improvement in an LTH grade II patient after 2 mo of PPI treatment, this patient should continue the PPI treatment continuously for up to $6 \mathrm{mo}$.

PPIs inhibit the maximal acid output when they are taken before a meal in the morning and in the evening [15]. However, there is no research suggesting a PRN PPI treatment for LPR patients. If a PPI treatment is continuous, the patient will spend more money for 6 mo of treatment.

\section{CONCLUSION}

The PPI treatment consisting of $30 \mathrm{mg}$ of lansoprazole twice daily for 6 mo improved the RSI scores during the first 2 mo and the second 2 mo. However, further PPI treatment for the third 2 mo did not provide any improvement. Overall, there was a significant difference between mean RSI scores before and after the PPI treatment. Additionally, both the continuous and PRN PPI treatments improved the clinical signs in the larynx and the surrounding area, as shown by the slow and continuous RFS decrease for up to 6 mo of PPI treatment.

The PPI treatment resulted in a decreasing degree of LTH. In those patients that are PPI resistant during the first $2 \mathrm{mo}$, the PPI treatment should continue through 4 mo for LTH grade II cases. For LTH grade III cases, other treatment options should be considered.

\section{LIMITATIONS}

The unavoidable limitation of this study was that not all of the eligible patients were willing to participate. Additionally, some of the patients were lost to follow-up during the intervention period. Including a larger number of patients in a similar study could help to make the results more generalizable and representative of this patient population.

\section{ACKNOWLEDGEMENT}

This article was presented at The $3^{\text {rd }}$ International Conference and Exhibition on Indonesian Medical Education and Research Institute (ICE on IMERI 2018). All authors read and approved the final manuscript. The contribution of each author, as follow; ST: study design, drafting, patient recruitment, data acquisition, data analysis, data interpretation, manuscript editing and accountability for all aspects of the research. HAAR: paper review and correction, data interpretation, final analysis, final approval, and accountability for all aspects of the research. AB: study design, data analysis, correlation analysis, drafting, and data interpretation.

\section{AUTHORS CONTRIBUTIONS}

All the author have contributed equally

\section{CONFLICT OF INTERESTS}

The authors declare there is no conflict of interest

\section{REFERENCES}

1. Chiba T. Laryngopharyngeal reflux disease (LPRD)-review article. Med Res Arch 2017;5:2-14.

2. Campagnolo AM, Priston J, Thoen RH, Medeiros T, Assuncao AR. Laryngopharyngeal reflux: diagnosis, treatment, and latest research. Int Arch Otorhinolaryngol 2014;18:184-91.

3. Belafsky PC, Postma GN, Koufman JA. The validity and reliability of the reflux symptom index (RSI). J Voice 2002;16:274-7.

4. Belafsky PC, Postma GN, Koufman JA. The validity and reliability of the reflux finding score (RFS). Laryngoscope 2001;111:1313-7.

5. Lechien JR, Huet $K$, Khalife M, Fourneau AF, Delvaux V, Piccaluga $\mathrm{M}$, et al. Impact of laryngopharyngeal reflux on subjective and objective voice assessments: a prospective study. J Otolaryngol Head Neck Surg 2016;45:59.

6. Reimer C, Bytzer P. Management of laryngopharyngeal reflux with proton pump inhibitors. Ther Clin Risk Manag 2008;4:225-33.

7. Sato K, Umeno H, Chitose S, Nakashima T. Tetra-probe, $24 \mathrm{~h} \mathrm{pH}$ monitoring for laryngopharyngeal reflux: a technique for simultaneous study of hypopharynx, esophagus and stomach. J Laryngol Otol Suppl 2009;31:117-22.

8. Chang BA, MacNeil SD, Morrison MD, Lee PK. The reliability of the reflux finding score among general otolaryngologists. J Voice 2015;29:572-7.

9. Ozturan O, Dogan R, Yenigun A, Veyseller B, Yildirim YS. Photographic objective alterations for laryngopharyngeal reflux diagnosis. J Voice 2017;31:78-85.

10. Habermann W, Schmid C, Neumann K, Devaney T, Hammer HF. Reflux symptom index and reflux finding score in otolaryngologic practice. J Voice 2012;26:e123-7.

11. Martinucci I, de Bortoli N, Savarino E, Nacci A, Romeo SO, Bellini $\mathrm{M}$, et al. Optimal treatment of laryngopharyngeal reflux disease. Ther Adv Chronic Dis 2013;4:287-301.

12. Pearson JP, Parikh S, Orlando RC, Johnston N, Allen J, Tinling SP, et al. Review article: reflux and its consequences--the laryngeal, pulmonary and esophageal manifestations. Conference held in conjunction with the 9th International Symposium on Human Pepsin (ISHP) Kingston upon Hull, UK, 21-23 April 2010. Aliment Pharmacol Ther 2011;33 Suppl 1:1-71.

13. Sataloff RT, Hawkshaw MJ, Gupta R. Laryngopharyngeal reflux and voice disorders: an overview on disease mechanisms, treatments and research advances. Discovery Med 2010;10:213-24.

14. Patigaroo SA, Hashmi SF, Hasan SA, Ajmal MR, Mehfooz N. Clinical manifestations and role of proton pump inhibitors in the management of laryngopharyngeal reflux. Indian J Otolaryngol Head Neck Surg 2011;63:182-9.

15. Shin JM, Sachs G. Pharmacology of proton pump inhibitors. Curr Gastroenterol Rep 2008;10:528-34. 